\title{
Flight Operations for the LCROSS Lunar Impactor Mission
}

\author{
Paul D. Tompkins* \\ Stinger Ghaffarian Technologies, Moffett Field, CA 94035 \\ Rusty Hunt ${ }^{\dagger}$, Matt D’Ortenzio ${ }^{\ddagger}$, James Strong ${ }^{\S}$, Ken Galal", John L. Bresina ${ }^{\#}$, Darin Foreman ${ }^{* *}$, Robert Barber ${ }^{\dagger \dagger}$, \\ Mark Shirley $\$$ \\ NASA Ames Research Center, Moffett Field, CA 90435 \\ James Munger ${ }^{\S \S}$, Eric Drucker ${ }^{\text {Tा }}$ \\ Northrop Grumman Aerospace Systems, One Space Park, Redondo Beach, CA 90278
}

\begin{abstract}
The LCROSS (Lunar CRater Observation and Sensing Satellite) mission was conceived as a low-cost means of determining the nature of hydrogen concentrated at the polar regions of the moon. Co-manifested for launch with LRO (Lunar Reconnaissance Orbiter), LCROSS guided its spent Centaur upper stage into the Cabeus crater as a kinetic impactor, and observed the impact flash and resulting debris plume for signs of water and other compounds from a Shepherding Spacecraft. Led by NASA Ames Research Center, LCROSS flight operations spanned 112 days, from June 18 through October 9, 2009. This paper summarizes the experiences from the LCROSS flight, highlights the challenges faced during the mission, and examines the reasons for its ultimate success.
\end{abstract}

\section{Introduction}

$\mathrm{T}$ HE LCROSS (Lunar CRater Observation and Sensing Satellite) mission was conceived as a low-cost means of determining the nature of hydrogen detected at the polar regions of the moon ${ }^{1}$. The mission presented several inherent challenges - planning for and achieving a precise impact at a lunar pole, guiding a Centaur upper stage far beyond its operational lifetime, co-launching as a secondary payload with the LRO spacecraft ${ }^{2}$, and preparing for and operating such a mission under a highly constrained budget and short development cycle. Beyond these, the flight itself presented other hurdles in the path to success, including several anomalies, one of which was nearly mission catastrophic.

The remainder of Section I provides the reader with background on the LCROSS project and components. Section II provides an overview of the flight mission. The remaining sections describe specific challenges encountered in planning and executing the mission and how they were overcome. The paper concludes by highlighting the key factors for the mission's success.

\section{A. The Project}

Managed, partially developed, and operated from NASA Ames Research Center (ARC), LCROSS was selected under the Exploration Systems Mission Directorate's (ESMD's) Lunar Precursor Robotic Program (LPRP) as a secondary payload manifested with the Lunar Reconnaissance Orbiter (LRO), under a $\$ 79$ million cost cap ${ }^{3}$.

\footnotetext{
${ }^{*}$ LCROSS Lead Flight Director, Code TI, Intelligent Systems Division, M/S 269-3

${ }^{\dagger}$ LCROSS Flight Director, Code TI, Intelligent Systems Division, M/S 269-3

${ }^{\ddagger}$ LCROSS Flight Controller, Code TI, Intelligent Systems Division, M/S 269-1

${ }^{\S}$ LCROSS Flight Controller, Code PX, Project Management Division, M/S 240A-3, AIAA Senior Member

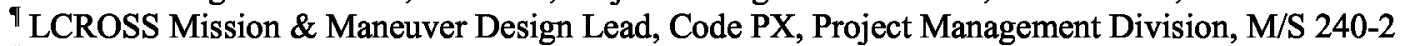

${ }^{\#}$ LCROSS Activity Planning \& Sequencing Lead, Code TI, Intelligent Systems Division, M/S 269-2

${ }^{* *}$ LCROSS Engineering Analysis \& Support Lead, Code RE, Engineering Systems Division, M/S 240A-3

${ }^{{ }^{\dagger \dagger}}$ LCROSS Project Systems Engineer, Code RE, Engineering Systems Division, M/S 240-2

${ }^{\ddagger}$ LCROSS Payload Engineer, Code TI, Intelligent Systems Division, M/S 269-2

$\$$ LCROSS Systems Engineer, System Definition Department, M2/2300

${ }^{7 \pi}$ LCROSS Systems Engineer, Space Vehicle Systems Engineering, M2/2300
}

1

American Institute of Aeronautics and Astronautics 
LCROSS was designated as a Class D mission, indicating NASA's willingness to accept greater levels of programmatic and operational risk.

Rather than incur the high cost and mission complexity associated with landing at a lunar pole, LCROSS precisely guided its Centaur Earth Departure Upper Stage (EDUS) to a lunar target as a kinetic impactor to raise a plume of polar regolith from the surface. After releasing the Centaur, the guiding Shepherding Spacecraft (S-S/C) employed a suite of nine science instruments to analyze the impact flash and plume materials at close range. Earthbased and orbiting assets, including LRO and the Hubble Space Telescope, acted in a supporting role for impact observations.

The LCROSS project remained on-budget and on-schedule, despite many programmatic and technical challenges. LCROSS launched with LRO on June 18, 2009, and on October 9, 2009, after 112 days in flight, the LCROSS team successfully delivered both the Centaur and S-S/C on-target within the Cabeus crater and collected extensive data from all instruments during and after the Centaur impact. After one month of post-impact analysis, the LCROSS science team announced the positive identification of water on the floor of Cabeus.

\section{B. The Shepherding Spacecraft}

In a novel design simplification, the LCROSS S-S/C was built around an EELV Secondary Payload Adapter (ESPA) ring, originally designed as a mating interface for up to six small spacecraft or experiments as secondary payloads on Delta IV and Atlas V launches ${ }^{4}$. Developed by Northrop Grumman, the S-S/C featured five equipment panels and a solar array mounted radially at the ESPA mate ports. A monopropellant tank was mounted within the ESPA ring. For launch, the nearly $900 \mathrm{~kg}$ (wet mass) LCROSS S-S/C was mated between the Centaur upper stage and LRO.

The S-S/C utilized copies of several LRO avionics units, including its RAD750-based single-board computer, communications card, and power and thruster control electronics. Onboard communications mixed SpaceWire and MIL-STD 1553 buses. LCROSS flight software derived heavily from software on previous programs, including EO-1 and WMAP. LCROSS communicated in S-band

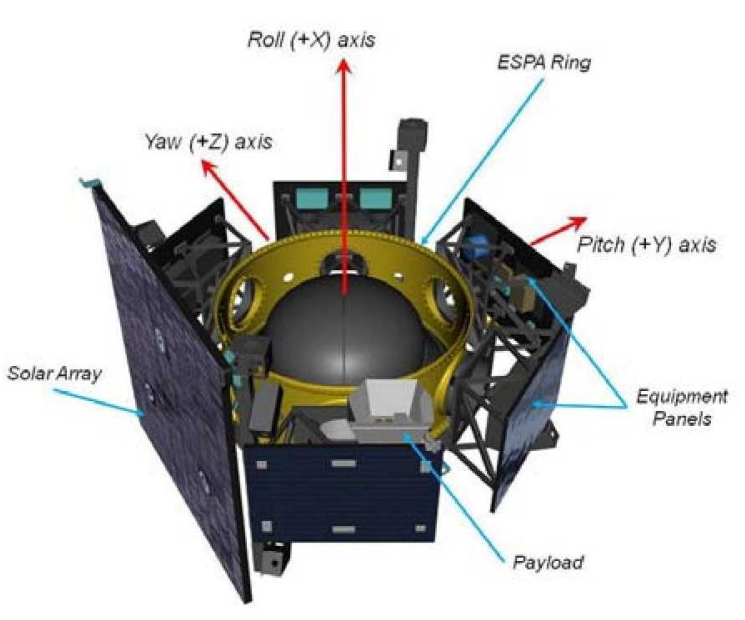

Figure 1. LCROSS Shepherding Spacecraft. The Centaur mated to the underside of the ESPA ring (in gold) via an adapter fitting. A second adapter fitting mated LRO to the top of the ESPA ring. The payload boresight is aligned with the Roll $(+X)$ axis, and the solar array normal is opposite the Pitch $(+Y)$ axis. via two omnidirectional antennas and two medium-gain antennas (20 deg half-angle). An S-band transponder enabled downlink at data rates up to $1 \mathrm{Mbps}$ and uplink at $4 \mathrm{kbps}$. Following launch and LRO separation, attitude control was passed from the Centaur to the S-S/C. The attitude control system (ACS) provided three-axis control using an inertial reference unit (IRU; no accelerometers), a star tracker, coarse sun sensors for emergency automated sun-relative attitude capture and tracking, and a set of eight monopropellant $5 \mathrm{~N}$ thrusters. Two additional $22 \mathrm{~N}$ thrusters provided orbit maneuvering capability. The ACS featured twelve control mode/submode combinations, six tailored for specific operations while attached to the Centaur, and a second set for use after Centaur separation. The LCROSS propellant tank contained just over $305 \mathrm{~kg}$ of hydrazine for both attitude control and orbit maneuvering. The LCROSS power system featured a $653 \mathrm{~W}$ solar array and four $20 \mathrm{~A}-\mathrm{h}$ batteries. LCROSS performed thermal control using a combination of resistive heaters, passive radiators, and multi-layer insulation.

\section{Science Instrument Payload}

The LCROSS science payload, developed at NASA ARC, combined processing and control electronics (Data Handling Unit, or DHU) with nine instruments to aid in water detection ${ }^{5}$ : a UV/visible light camera (VIS), two nearinfrared cameras (NIR 1 and 2), two mid-infrared cameras (MIR 1 and 2), a UV/visible light spectrometer (VSP), two near-infrared spectrometers (NSP), and a total luminescence photometer (TLP). The DHU, all instruments, and optics were COTS products or incrementally adapted from COTS products under a $\$ 2.5$ million budget. Eight of nine instruments were co-aligned along the $\mathrm{S}-\mathrm{S} / \mathrm{C}$ longitudinal axis and provided nadir-pointed sensing during the Centaur impact event. One of two near-infrared spectrometers was side-pointed to provide spectra of sunlit material 
rising in the Centaur ejecta plume. A spring-loaded cover protected the nadir-looking instruments from direct sun exposure during launch and through the early part of the mission.

\section{The Centaur}

The Centaur Earth Departure Upper Stage (EDUS), developed by United Launch Alliance, utilized two independent propulsion systems. A cryogenic liquid hydrogen and liquid oxygen system feeding the Centaur main engine boosted LCROSS and LRO into Low-Earth Orbit (LEO) and performed Trans-Lunar Injection (TLI). A separate hydrazine-based propulsion system enabled attitude control until handover to the LCROSS S-S/C. Onboard processing controlled all Centaur maneuvering, initiated the LRO separation, and issued discrete commands that triggered LCROSS activation. A post-TLI command sequence was designed to deplete most propellants from both systems before control handover to LCROSS. The only significant physical modification of the LCROSS Centaur was in the application of white paint to aid in thermal balancing of the empty stage during the mission. The Centaur mass was approximately $2400 \mathrm{~kg}$ at impact. See Ref. 6 for a detailed treatment of LCROSS Centaur operations.

\section{E. Mission Operations}

LCROSS mission operations were conducted primarily at NASA ARC from a seven-seat Mission Operations Control Room, a Mission Support Room for planning and off-line analysis, and a Science Operations Center. The ARC team was supported by several remote operations facilities and personnel at the Jet Propulsion Laboratory (navigation and link scheduling), NASA Goddard Space Flight Center (maneuver design support) and two Northrop Grumman sectors (real-time and off-line engineering support). The flight team was divided into two shifts of realtime spacecraft support, and another team devoted mainly to maneuver design, communications scheduling, and engineering analysis. For details on the LCROSS flight team, please consult Tompkins et al. (Ref. 7).

LCROSS utilized the NASA/JPL Deep Space Network for space-ground communications. Uplink communications were exclusively via $34 \mathrm{~m}$ antennas. Downlink occurred primarily through $34 \mathrm{~m}$ antennas, but also through $70 \mathrm{~m}$ antennas (for high-rate science downlink applications) and the $26 \mathrm{~m}$ antenna at Canberra (for initial acquisition). At the time of launch, the Madrid $70 \mathrm{~m}$ antenna (DSS-63) was down for upgrades, but did serve in an engineering test capacity to support several events. Details on communications performance appear in Ref. 8.

\section{Summary of Mission Events}

The LCROSS mission was focused on achieving a precise, high-energy Centaur impact within a lunar polar crater, and on observing the impact and resulting ejecta plume from the S-S/C, other orbiting spacecraft, and Earthbased observatories. To achieve the desired impact geometry, LCROSS performed a lunar gravity assist to transfer from its trans-lunar orbit to a Lunar Gravity Assist Lunar Return Orbit (LGALRO), an elliptical, Earth-centered orbit of roughly lunar semi-major axis, but inclined steeply with respect to the lunar orbit plane. Performing a series of trajectory correction maneuvers (TCM's) with the Centaur impactor still attached, the S-S/C gradually refined the trajectory to hit the designated target. Following three revolutions of the Earth, the LCROSS and lunar orbits reintersected. Less than ten hours before impact, the S-S/C released the Centaur. Soon afterward, a braking maneuver induced a delay between the Centaur and S-S/C impacts, allowing the S-S/C to capture and transmit data from the Centaur impact event for four minutes before creating a secondary impact plume nearby. This was the primary LCROSS science operation. However, over the mission, the payload was activated eight additional times to collect engineering and instrument calibration data, and another five times to manage instrument command sequences.

The LCROSS mission can be described as a sequence of four operational phases: Launch (liftoff to S-S/C activation), Transfer (activation to lunar gravity assist), Cruise (gravity assist through TCM 8), and Final Targeting and Impact (TCM 8 through S-S/C impact). This section reviews each of these mission phases.

\section{A. Launch Phase}

LCROSS and LRO launched on June 18, 2009 at 21:32 UTC aboard an Atlas V launch vehicle in the 401 configuration (4-meter fairing, no solid rocket boosters, single-engine Centaur). After achieving low-Earth orbit, the Centaur performed a 23 minute coast, and then re-ignited to perform the trans-lunar injection (TLI) burn for both LCROSS and LRO. Just under 3 minutes after TLI, the Centaur separated from LRO and subsequently performed a collision and contamination avoidance maneuver. Having delivered LRO, the Centaur performed a series of additional blowdown maneuvers totaling approximately $31 \mathrm{~m} / \mathrm{s}$ to place LCROSS closer to its desired transfer orbit. To minimize the residual propellant species aboard the Centaur at lunar impact (potential contaminants of water measurements), the Centaur next performed a sequence of propellant depletion maneuvers with both cryogenic and hydrazine-based reaction control systems. 
LCROSS launched powered off (see Section IV.A). Roughly 30 minutes after LRO separation, the Centaur initiated spacecraft activation via a series of six discrete commands, issued over 14 seconds, to trigger primary or redundant stages of the power-up. These included connecting batteries and solar array safely to the power bus; booting the spacecraft processor; initiating active thermal control; powering the spacecraft transponder to initiate telemetry downlink; and opening a latch valve to fill propellant lines. Importantly, these first six discrete commands left LCROSS under Centaur attitude control. As the S-S/C proceeded with activation, the Centaur oriented the LCROSS solar array toward the sun to maintain power and thermal control, and continued venting propellants. Meanwhile, with the spacecraft avionics and transponder activated, the mission operations team began a basic spacecraft checkout (telemetry analysis, commanding, downlink rate changes, basic configuration setup). Triggered by the depletion of Centaur propellants (between 88 and 105 minutes after discrete 1), the Centaur issued a final discrete command to cede attitude control to the S-S/C. This final command prompted the S-S/C to enter Sun Point Mode, an attitude control mode that pointed the S-S/C solar array toward the sun (enforcing benign power and thermal conditions) and maintained a $0.1 \mathrm{deg} / \mathrm{s}$ rate about the solar array normal axis (S-S/C pitch axis).

The Centaur remained active for hours after handover to transmit Centaur telemetry, including tank pressures and temperatures, to aid in the estimation of remaining Centaur propellant.

\section{B. Transfer Phase}

Beginning at attitude control handover, Transfer Phase spanned the four days required to transit from the Earth to the moon, including the lunar gravity assist. The principal goals of Transfer Phase were to confirm spacecraft health, to refine the trajectory for the lunar encounter, and to perform a number of payload calibrations. Per plan, Transfer Phase was conducted under nearly 24 -hour communications coverage by the DSN. The fast operational pace prompted a 24-hour staffing schedule utilizing two overlapping 13-hour fully staffed shifts.

Immediately after handover, the flight team configured LCROSS for Cruise State, including a transition from Sun Point Mode to Stellar Inertial Mode, allowing LCROSS to be oriented to an inertially-fixed, sun-pointed, ecliptic-normal attitude. The flight team also continued spacecraft checkout, with transitions to all ACS attached control modes, performance of several small $(<15 \mathrm{deg})$ slew maneuvers, and a small delta-v maneuver (this and all other delta-v maneuvers are summarized in Table 1). A Centaur gas leak (section V.A) and a number of spacecraft anomalies (sections V.B and V.C) were discovered during checkout.

During Transfer Phase, the flight team performed a first TCM 25 hours after LRO separation to correct for Centaur delivery

Table 1. Summary of Delta-V Maneuvers. TCM 1 and $5 a$ were "deterministic" burns required even under ideal conditions.

\begin{tabular}{|l||c||c||c|}
\hline Maneuver & Start UTC & Duration (s) & Magnitude (m/s) \\
\hline \hline DV Test & & & 0.210 \\
\hline \hline TCM 1 & 19 June 2009 23:15 & 579.6 & 8.060 \\
\hline TCM 2 & 20 June 2009 20:16 & 112.6 & 1.502 \\
\hline TCM 3 & 22 June 2009 10:30 & 64.0 & 0.862 \\
\hline TCM 4a & cancelled & -- & -- \\
\hline TCM 4b & cancelled & -- & - \\
\hline TCM 5a & 21 July 2009 13:00 & 1790.0 & 21.124 \\
\hline TCM 5b & cancelled & -- & -- \\
\hline TCM 5c & cancelled & -- & -- \\
\hline TCM 6 & cancelled & -- & - \\
\hline TCM 7 & 5 Sep 2009 11:30 & 39.4 & 0.324 \\
\hline TCM 8 & 30 Sep 2009 15:00 & 45.8 & 0.351 \\
\hline TCM 9 & 6 Oct 2009 00:00 & 7.6 & 0.055 \\
\hline TCM 10 & cancelled & -- & -- \\
\hline Separation & 9 Oct 2009 01:50 & 0.0 & 0.154 \\
\hline Braking & 9 Oct 2009 02:30 & 240.0 & 9.030 \\
Burn & & & \\
\hline
\end{tabular}

errors and to target Lunar Swingby orbit conditions. Two additional TCM's were performed, 49 and 84 hours after LRO separation, to correct errors induced by earlier burns. The LCROSS spacecraft behaved nominally during all three maneuvers, putting the spacecraft on target for the Lunar Swingby event.

Four hours after TCM 1, the payload was powered-up in flight for the first time in what was termed "Quicklook". Performing a short diagnostic with each instrument (save the total luminescence photometer, which was not powered-up), the Payload Engineer and Science Team determined that the payload was behaving nominally.

Just over five hours after TCM 3, the team performed a second payload activity, "Star Field Calibration", during which the payload instrument cover was opened, and images were taken of a known star field. From these measurements, the Science Team estimated camera alignment offsets with respect to the star tracker reference frame. These offsets closely matched pre-launch estimates and consequently the original offsets were used as the basis for Lunar Swingby planning.

The culmination of Transfer Phase was the lunar gravity assist event on Day 5, called "Lunar Swingby". The goals of Lunar Swingby were twofold: first, to efficiently transfer the LCROSS spacecraft into its LGALRO cruise trajectory; second, to take advantage of the only close approach to the moon (save impact) to gather higherresolution science measurements of the lunar surface and limbs for calibration purposes. Because of its focus on science operations, similar operational structure, use of a $70 \mathrm{~m}$ DSN antenna at a $1 \mathrm{Mbps}$ downlink rate, and time- 
critical nature, the event also served as a dress rehearsal for the impact event. In practice, Lunar Swingby partially overlapped operations supporting LRO's mission-critical lunar orbit insertion (LOI) burn. This introduced complexities to the communications link schedule and risk to Lunar Swingby, but did not ultimately adversely affect either mission (see section IV.B). Data from Lunar Swingby were instrumental in detecting issues with image compression (see section V.F) and exposure settings. Changes to payload command sequences and operational procedures stemming from these findings were critical to the success of impact observations.

\section{Cruise Phase}

The bulk of the LCROSS flight was spent in Cruise Phase, which began at the conclusion of Lunar Swingby, and ended at TCM 8, just 9 days before lunar impact. The LGALRO was designed to have LCROSS re-encounter the moon after three revolutions about the Earth, each of roughly 36 days. Under idealized trajectory planning, Cruise Phase entailed only one TCM to target desired impact position, geometry, and timing parameters. However, the mission plan incorporated additional TCM's to correct errors stemming from the lunar gravity assist and subsequent TCM's; to compensate for unmodeled orbit perturbations; and to respond to in-flight refinements of the target Centaur impact site ${ }^{* * *}$. Cruise Phase also presented opportunities for additional payload calibrations and dictated several engineering events, including periodic spacecraft reorientations to re-point the primary omni-directional antenna toward Earth, activities to remove ice from the Centaur exterior, and additional checkout activities. After the operational intensity of Transfer Phase, Cruise Phase was designed to scale back on DSN contacts to an average of one track of 4 to 6 hours duration every three days, greatly reducing the staffing required to support the mission.

The early part of the first Cruise orbit was devoted to transitioning to intermittent DSN coverage. Importantly, the anomalous thruster thermal behavior discovered early in Transfer Phase (section V.B) required in-flight modifications to onboard fault management, ground command sequences, and operational procedures before the flight team was comfortable leaving the spacecraft unmonitored for long periods. Post-Lunar Swingby trajectory errors were too small to justify executing the planned TCM $4 \mathrm{a}$ and $4 \mathrm{~b}$ maneuvers. The first Cruise TCM executed was TCM 5a, the second and final deterministic burn for the mission, conducted on Day 33. TCM 5a was executed very accurately, enabling the team to skip TCM 5b, TCM 5c, and TCM 6 on subsequent revolutions.

On Day 26, before TCM 5a, LCROSS conducted its first "Cold Side Bake" event in an attempt to remove ice from the Centaur exterior (section V.D and Table 2). A second Cold Side Bake event was added to the schedule and executed 15 days later. Dissatisfactory results from this second attempt ultimately justified the insertion of yet another bakeout event during the second Cruise Phase orbit.

The second Cruise Phase orbit presented two opportunities for science instrument calibrations. The first, "Earth Look 1", swept the instrument boresights across the Earth disk on Day 45 for enhanced camera alignment calibration and spectral calibrations on a well-understood target. The second opportunity, executed on Day 60, was identified after launch, and enabled a simultaneous view of the crescent Earth and moon. This "Earth/Moon Look Calibration" was added to test approaches for removing MIR2 instrument blur, which was noticed during previous calibrations, possibly due to slippage of the lens during launch. Both calibration events executed nominally and provided additional data to guide refinements to the impact sampling strategy.

On the second half of the second Earth orbit, as discovered immediately before the scheduled third Cold Side Bake event, LCROSS experienced a major propellant loss stemming from a short-lived IRU glitch (see section V.E). With the mission in jeopardy, LCROSS declared an emergency and transitioned to maximum DSN coverage to enable full-time monitoring. Over the following two weeks, the flight team worked to develop strategies to prevent another excessive propellant usage. With operational and software protections in place, the LCROSS project returned to a nominal operational posture on Day 78, albeit with a significantly smaller propellant margin.

During the third and final Earth orbit, all activities were focused on preparations for impact. For training purposes, the team conducted a series of nominal and contingency rehearsals of the final day's activities, interleaved with actual spacecraft monitoring and activity support. Earth Look Calibration 2 and Moon Look Calibration, both scheduled for the final orbit, were victims of propellant-saving measures and cancelled. However, a reduced-scope "Earth Gaze Calibration", designed to use far less propellant at the expense of some science return, was added and performed on Day 92. Its purpose was to re-confirm the operability of all instruments prior to impact.

Having cancelled the third Cold Side Bakeout due to the propellant loss, a replacement was planned and executed on Day 98 (see Table 2). In a propellant-saving measure, and owing to coincidentally ideal geometry, this activity was combined with the first on-orbit test of the minus- $Z$ axis Medium Gain Antenna (MGA), the antenna designated for the impact data downlink. The MGA test was successful; bakeout results were inconclusive.

\footnotetext{
*** In anticipation of late-arriving lunar data from LRO and other lunar missions, the LCROSS mission design allowed the Science Team to select the final impact target within 30 days of impact without significant penalty.
} 
In the final two weeks of flight, the Science Team made two refinements of the impact target position: before TCM $7(\Delta=97 \mathrm{~km})$, and between TCM 8 and $9(\Delta=9.2 \mathrm{~km})$. Each update resulted in changes to the following burn plan to remove the remaining position error. TCM 7 (Day 99) and 8 (Day 104) were executed nominally to remove estimated pre-burn impact position errors of roughly $520 \mathrm{~km}$ and $44 \mathrm{~km}$, respectively.

\section{Final Targeting and Impact Phase}

To support precise navigation and burn planning prior to impact, this phase re-introduced 24-hour DSN tracking, and added tracking performed by the Applied Physics Laboratory. TCM 9 and 10 remained in the mission plan to refine the impact position and time of the Centaur impact. TCM 9 was designed to remove the error $(9 \mathrm{~km})$ introduced primarily by the change in target position; it executed nominally (Day 111), reducing the predicted impact error to $950 \mathrm{~m}$ (requiring a correction of $15 \mathrm{~cm} / \mathrm{s}$ ). The maneuver design team determined that the delta-v induced by separation could be used to precisely nudge the Centaur into the desired impact trajectory. Consequently, the team waived TCM 10, avoiding the added complexity and greater performance uncertainty of another burn.

Centaur separation was performed successfully 9 hours 40 minutes prior to Centaur impact and, with the substantial change of mass properties, was accompanied by a transition to a completely new set of attitude control modes tuned for post-separation conditions (and untested in flight). One minute following separation, the S-S/C flipped 180 degrees to point the payload at the receding Centaur. The spacecraft payload was activated to transmit imagery of the Centaur for 15 minutes (via $70 \mathrm{~m}$ DSN antenna), to determine whether the separation had caused the Centaur to tumble. Forty minutes after separation, the S-S/C performed the Braking Burn, a delta-v maneuver used to induce a four-minute delay between the Centaur and S-S/C impact events $(598 \mathrm{~km}$ range at Centaur impact).
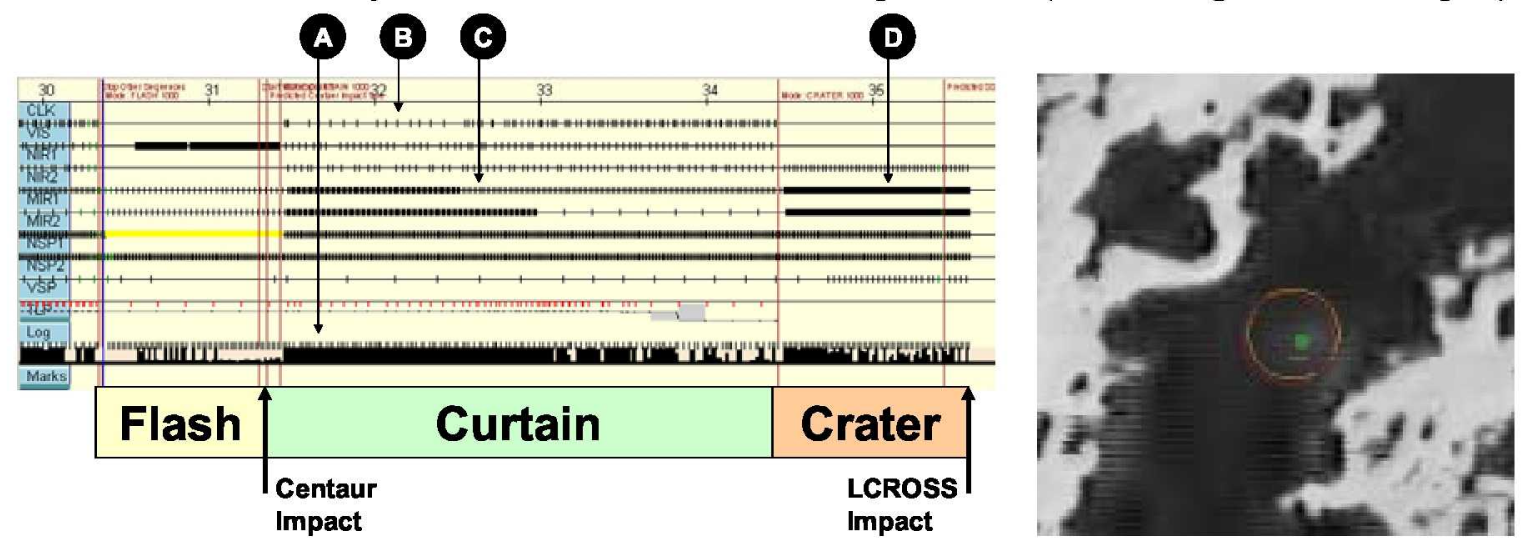

Figure 2. Impact Science Observations. The left diagram is a timeline of instrument data collection covering the five minutes prior to $S$-S/C impact. Label " $A$ " shows downlink fully subscribed; " $B$ " shows irregular ticks that reflect images dropped onboard due to downlink over-subscription; " $C$ " shows the effect of a ground command sent to reduce camera sampling rates; " $D$ " shows how a command sent to change an image exposure setting enabled imaging the Centaur crater in near-infrared. The right image is the NSP 1 field-of-view just after Centaur impact.

The flight team re-planned the impact sequence using post-burn orbit estimates. In the hours before Centaur impact, the team disabled a large fraction of onboard fault management to prevent faults from interrupting impact observations, and acquired the $70 \mathrm{~m}$ and two $34 \mathrm{~m}$ antennas at Goldstone (downlink via MGA at $1 \mathrm{Mbps}$ ). The impact command sequence steered the S-S/C attitude to keep the payload boresight pointing toward the intended Centaur impact point while pointing the MGA toward Earth, under an ACS mode that enforced a 0.1 deg deadband. The payload was activated a final time 56 minutes prior to Centaur impact, during which the science and payload team confirmed payload operability, including the only in-flight power-up of the total luminescence photometer.

Impact observations were divided into three phases: Flash, Curtain, and Crater. Figure 2 depicts the data return from each science instrument (one row for each; one tick for each image or spectrum) during the final five minutes of flight. Flash Phase, which began one minute prior to the expected Centaur impact time, was designed to capture the light released at impact. The nadir-looking near-infrared spectrometer (NSP1) was commanded to a high speed, low spectral resolution mode (shown in yellow). The mid-infrared cameras (MIR1/2) were triggered to observe the impact in one image each, while the total luminescence photometer sampled at $1000 \mathrm{~Hz}$. Curtain Phase, designed to capture the evolution of the ejecta cloud, emphasized recording near-infrared and ultraviolet/visible spectra (NSP1 and VSP instruments respectively). Crater Phase emphasized imaging the Centaur crater with thermal cameras (MIR1/2). The image at the right of Figure 2 shows the NSP1 field of view (pre- and post-Lunar Swingby alignment 
estimates), superimposed on an image of Cabeus. The green dot is the estimated location of the Centaur impact from thermal imagery taken seconds before S-S/C impact. Image compression artifacts (see section V.F) are visible in the lower left. Pre-publication estimates place the terrain-relative Centaur impact position error at $75 \mathrm{~m} \pm 82 \mathrm{~m}$.

\section{Pre-Launch and In-Flight Mission Planning}

Trajectory and maneuver planning to achieve a precise impact was a particularly challenging aspect of the mission. The problem balanced meeting very specific impact and observation conditions against the limitations of the spacecraft - perhaps most importantly its finite propellant load. A complex design cycle and several launch date slips made this an exceptionally work-intensive part of LCROSS development. In flight, a number of natural and self-induced orbit perturbations counteracted efforts to achieve precision. As so much was being learned about the lunar poles even after launch, the impact target was re-specified repeatedly until 9 days before impact.

\section{A. Impact Requirements}

LCROSS satisfied numerous requirements governing the accuracy, geometry and timing of its impact, including:

1) To enable impact at either lunar pole (latitudes $>70 \mathrm{deg}$ ) with a targeting accuracy goal of $+/-1.75 \mathrm{~km}$;

2) To achieve an impact angle (between the impact velocity vector and the crater floor) of no less than $60 \mathrm{deg}$, but as close to $90 \mathrm{deg}$ as possible to maximize the height of the impact plume;

3) To impact the moon at a time such that a) the moon was oriented to tilt the impact point surface normal toward the Sun (to maximize lighting of the ejecta) and Earth (to maximize visibility from Earth); b) the moon phase was more than $30 \mathrm{deg}$ from new moon or full moon; c) the moon was simultaneously visible from the DSN, Hawaii, the Hubble Space Telescope (HST) and LRO, and d) the prime observatory in Hawaii was in nighttime conditions with a minimum of two hours after dusk and before dawn.

Within a month of impact, the final selection of the impact site was made, with a target chosen deep inside Cabeus crater (lon $311.302 \mathrm{deg}$, lat $-84.674 \mathrm{deg}$ in lunar Mean Earth rotating coordinates). While this choice of impact target increased the likelihood of detecting water by LCROSS, the depth of the site $(3.8 \mathrm{~km}$ below the mean lunar radius) made it more difficult for Earth-based observatories to acquire measurements of the impact plume. The final LCROSS impact time (10/9/09 11:31:19 UTC) was set in the final weeks and days prior to impact to permit optimal on-orbit observations from HST, and the LRO spacecraft coordinated a series of orbit maneuvers that allowed LRO to fly by the impact site shortly after impact.

\section{B. Trajectory Design Strategy}

LCROSS was required to minimally affect the LRO mission as a precondition to its selection as a secondary mission.

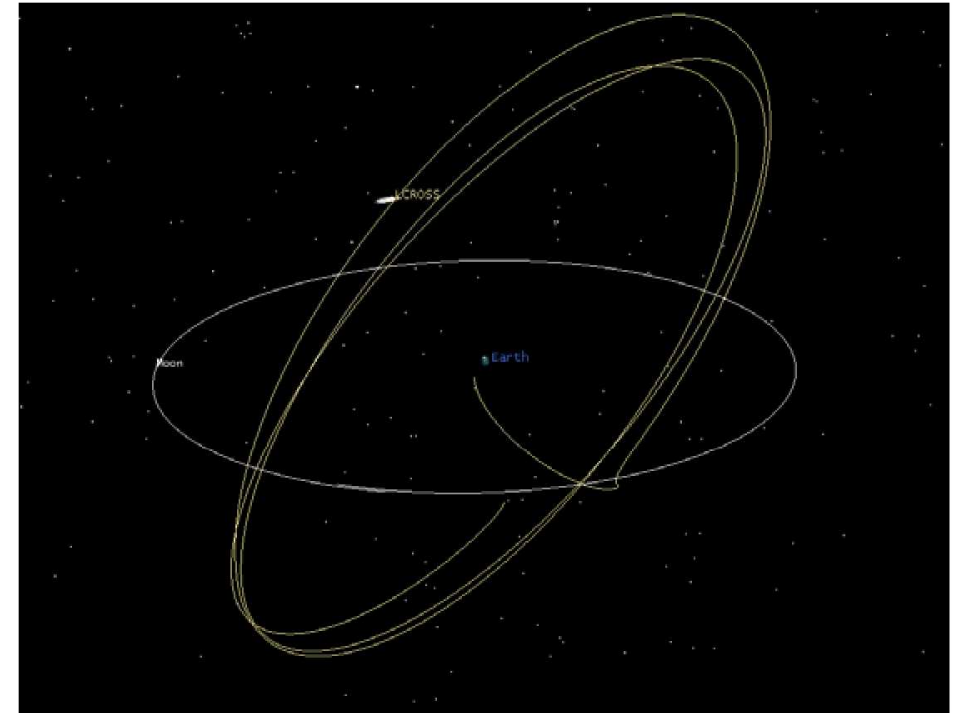

Figure 3. LCROSS Trajectory. In mission planning, varying the flyby direction and number of Earth revolutions provided flexibility to meet the impact requirements. As flown, the mission combined a south pole lunar flyby and three revolutions about Earth prior to a south pole impact (as shown above).

This necessitated that LCROSS be able to accommodate as many candidate LRO launch dates as possible. Given the above strict requirements, trajectories with variable mission durations had to be devised that could be phased with lunar libration cycles. The solution was to permit either south or north pole lunar gravity assists (governing whether impact occurred on an even number of moon revolutions after Lunar Swingby), to vary the lunar fly-by altitudes, and to vary the total number of Earth revolutions in order to establish the necessary LGALRO phasing trajectories. These trajectories resulted in Cruise Phase orbits with perigee at lunar distance and apogee set at varying distances that could sustain a sufficient number of revolutions about the Earth and impact the desired lunar pole during windows of opportunity that satisfied LCROSS impact requirements. Trajectories requiring between 2 to 5.5 revolutions of the Earth (with corresponding mission durations of 2.5 to 7 months) were designed to supported each of the 3-4 day LRO launch blocks that occurred every two weeks between April and July 2009. 
Per agreement with United Launch Alliance (ULA), LCROSS could rely on up to $50 \mathrm{~m} / \mathrm{s}$ of delta-v from the launch vehicle (following LRO separation) to help target its desired fly-by conditions. Since the launch vehicle injection burn targeted an LRO lunar insertion over the south pole $(\sim 350 \mathrm{~km}$ altitude), LCROSS trajectories that required a south pole Lunar Swingby could be directly targeted by the launch vehicle. For LCROSS trajectories requiring a north pole Lunar Swingby, the $50 \mathrm{~m} / \mathrm{s}$ launch vehicle contribution needed to be supplemented by a deterministic LCROSS maneuver (TCM 1) to complete the placement of periselene several thousand kilometers over the north pole.

At the time of final delivery of LCROSS launch targeting states to ULA, a north pole impact was baselined by the LCROSS science team. However, in April 2009, newly obtained lunar observation data prompted the science team to request a switch to a south pole impact. For the June 18 launch date, the change involved retargeting from a 3.5-month south pole swingby trajectory with a north pole impact to a 4-month south pole swingby trajectory with a south pole impact. Since in both cases a south pole swingby was required, a deterministic LCROSS maneuver of only $6 \mathrm{~m} / \mathrm{s}$ was needed to retarget the desired fly-by conditions.

\section{Orbit Perturbations and a Moving Target}

Trajectory planning was complicated by numerous natural orbit perturbations, including solar radiation pressure and accelerations induced by the sublimation of ice from the Centaur (see section V.D). Other perturbations were self-induced. Attitude control was performed exclusively via thrusters. Despite a design that "balanced" thruster firings to minimize orbit effects, second-order effects were still significant. Furthermore, for greater propellant efficiency, the team resorted to paired (unbalanced) thruster firings in response to the propellant demands of a Centaur gas leak and the propellant loss caused by an in-flight anomaly (detailed in sections V.A and V.E, respectively).

Knowledge of the impact target region on the lunar south pole increased dramatically during flight. Other active missions (Kaguya, Chandrayaan-1, and especially LRO) produced a host of data relating to suspected water concentration, crater depth (and view angle to Earth) and topography that transformed the LCROSS science team's targeting strategy. The LCROSS impact target was changed four times in flight, the last only 9 days before impact, following TCM 8. Each target update entailed maneuver re-planning, adding to the team's workload.

\section{Co-Manifestation and Concurrent Flight with LRO}

Beyond sharing the ride to space, co-manifestation with LRO on the Atlas V launch vehicle had an enormous influence on LCROSS. Though LRO imposed strict limitations on the LCROSS design and partially restricted its use of the DSN in the first week of operations, the LRO team provided invaluable support to LCROSS for impact site selection and conducted pre- and post-impact observations in support of the LCROSS science mission. The close collaboration that began between the two teams during spacecraft development continued successfully in mission planning and in flight.

\section{A. Power-Off Launch and Activation}

As a secondary payload launching with LRO, LCROSS, with its risk-tolerant Class D posture, was required to follow a policy of non-interference with the LRO mission that governed many aspects of the LCROSS program and spacecraft design. Operationally, this policy manifested itself in the design of LCROSS to be powered off while attached to LRO and to be activated autonomously after LRO separation. This requirement added a degree of complexity to the LCROSS spacecraft and operational designs.

On the launch pad, a minimal set of LCROSS telemetry, including battery voltages and the states of relays critical to spacecraft activation, was available to the launch site engineering support team via a data umbilical. These were a focus of launch commit criteria to prevent launching a malfunctioning LCROSS. At liftoff the umbilical was severed, leaving the flight team without spacecraft health data during ascent.

Section II.A describes the operations surrounding S-S/C activation. The S-S/C activation sequence, implemented in the Autonomy \& Fault Management subsystem, shared many of the same command sequences designed to place LCROSS into its "safe mode." It was designed to power the spacecraft and to place it in a benign configuration, without human intervention. The fault management design, and the flight team procedures to monitor them, evolved over many months and required significant testing and training.

\section{B. DSN Resource Contention and Resolution}

Following LRO separation, LCROSS and LRO maintained very similar moon-bound trajectories and shared the DSN as a primary communications network for critical events. LRO no longer imposed its non-interference 
requirement on LCROSS, and the two were treated as independent missions. Prior to launch, the two projects coordinated closely to resolve periods of DSN contention that were anticipated during their simultaneous transfers and lunar encounters. Based on technical discussions and considering event criticality for each mission, LRO updated the timing for its first Mid-Course Correction (MCC 1) to allow LCROSS to perform its first TCM as originally scheduled, with dual DSN antenna coverage. However, the criticality of LRO's Lunar Orbit Insertion burn (as compared to the LCROSS Lunar Swingby event, which was highly important but not mission-critical), prompted the teams to design a detailed DSN antenna handoff plan (different for each launch time) that would ensure the greatest chance of LOI success, while providing sufficient DSN coverage of LCROSS's Lunar Swingby in most cases. It is worth mentioning that in some scenarios, including for the actual launch date, the success of the Lunar Swingby hinged on the earlier success of the LRO LOI. In practice, the plan for the June 18 21:32 launch time provided DSN uplink and downlink time for LCROSS to upload commands for Lunar Swingby prior to the LRO LOI, and to capture all data from Swingby after the LOI, albeit at a higher-than-desired observation altitude.

\section{LRO Support of LCROSS Target Selection and Impact Observation}

The LRO team afforded LCROSS a tremendous advantage when it conducted a series of lunar south pole overflights and provided the project with data that was critical to the in-flight selection of the impact site. Perhaps more importantly, the selection of Cabeus as the impact site reduced the viability of using Earth-based observatories in support of plume detection and analysis. As a consequence, data taken on LRO's overflight of the LCROSS impact site after the Centaur impact became the only strong supplemental evidence in the discovery of water.

\section{Anomalies and Surprises}

The LCROSS team experienced a number of flight system anomalies and unexpected behaviors during the mission, but either fully recovered from them or significantly reduced their associated risk by introducing system modifications and operational workarounds. The following sections describe the detection and responses for six noteworthy off-nominal conditions.

\section{A. Centaur Gas Leak}

Analysis of the Centaur provided by ULA in the months before launch exposed a potential for residual propellants to leak from one or more valves on the spent stage for weeks following launch. Leaking gasses were predicted to induce a torque on the LCROSS stack which would be counteracted by S-S/C attitude control. Depending on the torque direction, magnitude and dissipation profile (as a function of the quantity of trapped propellants, the valve location, the leak rate, and leak effective thrust parameters), analysis showed that the resulting LCROSS propellant usage could range from inconsequential to catastrophic $(>100 \mathrm{~kg} /$ day). In the weeks immediately prior to launch, the LCROSS project developed operational strategies for assessing leak severity and counteracting leak effects to the degree possible. Furthermore, at the LPRP program level, the risk posed to the LCROSS mission by the Centaur leak (and the minimal time to respond to the information) influenced the decision to slip the LRO/LCROSS launch from the June 2 to the June 17 launch block (much higher propellant margin).

In the first day of flight, the team performed two torque observations, each three to four minutes long, during which attitude control was disabled to permit an observation of the attitude time history without thruster firings. These data were used to estimate the torques being experienced by the spacecraft. The flight team also observed the deadband behavior while under control and estimated propellant usage due to attitude control thruster firings. The team concluded that Centaur gasses were inducing a significant torque on the combined stack. On Day 1, LCROSS exhibited ACS propellant usage of approximately $6.5 \mathrm{~kg} / \mathrm{day}$ ( $>10$ times the predicted non-leak value). To minimize propellant usage, the team configured the spacecraft to fire thrusters in pairs instead of nominal quads. This removed the cancellation effect of balanced four-thruster sets, but caused all ACS firings to perturb the LCROSS orbit. The team also increased the ACS impulse bit from $50 \mathrm{~ms}$ to $100 \mathrm{~ms}$, thereby improving the effective specific impulse for typically short thruster firings. Over the flight, the torque direction changed, occasionally in inexplicable discrete jumps. Because these effects were only indirectly observed, it was never determined which Centaur valves may have been leaking, or whether there were other effects in play (e.g. ice sublimation from Centaur skin). Propellant usage for background ACS shrank to below $1.0 \mathrm{~kg} /$ day in the first week and to $0.25 \mathrm{~kg} /$ day by midmission. The propellant margin associated with the June 18 launch date was sufficient to absorb this cost.

\section{B. Thruster Thermal Control}

On Day 1 of the flight, spacecraft engineers observed that valve heaters for two $5 \mathrm{~N}$ thrusters were not activating at the measured valve low-temperature thresholds, thereby risking hydrazine freezing and propellant line rupture. 
LCROSS thruster valve heaters were controlled via fixed-setpoint thermostats attached to the thruster mounting brackets. The flight team later determined the cause to be a combination of insufficient thermal conductivity between the thruster valves and thermostats and excessive thermal conductivity between the thermostats and thruster nozzles and catalyst bed heaters. Cold thruster valve temperatures were not perceived by the thermostats, which were being warmed by repeated thruster firings and constant heat from the catalyst beds. The potential for this condition was discovered during spacecraft thermal vacuum testing and partially mitigated. However, due to project limitations, the problem could not be fully re-analyzed or re-tested until flight.

The operational effects of this anomaly were significant. Without direct control of thruster valve heaters, the flight team spent the first several days enacting various measures to warm the valves, including ground-commanding thruster firings, biasing the spacecraft attitude to warm the valves via direct exposure to sunlight, and brief entry to small deadband control submodes to induce frequent thruster firings. Despite their short-term warming benefit, frequent thruster firings, induced partially by the Centaur gas leak (see above), prevented the thruster brackets from reaching thermal equilibrium with the thruster valves. The final mitigation strategy combined modifications to operational practice and to fault management software tables. First, the LCROSS Cruise attitude was modified by adding a minus 20 degree bias in yaw such that the offending thruster valves were warmed by direct sun exposure. Second, onboard fault management was augmented to automatically trigger short sequences of thruster firings upon reaching the low valve temperature thresholds. The yaw bias was sufficient to control valve temperatures in all but a few specific cases, and hence the thermal issue did not become a burden on propellant. By experiment, the yaw bias was deemed necessary to control valve temperatures, and it was used until just before impact.

\section{Transponder Doppler Bias}

On the first day of flight, the Navigation team noticed a distinct $0.7 \mathrm{~Hz}$ bias in Doppler measurements below a specific signal frequency. This corrupted tracking measurements and prevented accurate estimates of the LCROSS trajectory, critical to precision targeting for full mission success. This problem was later attributed to a coding error in an FPGA within the transponder unit and not repairable in flight. To work around the issue, LCROSS requested that the DSN increase their uplink frequency by $5 \mathrm{kHz}$, thereby biasing the frequency above the threshold at which problems had occurred. This removed the perceived Doppler bias and all negative effects on orbit estimation.

\section{Centaur Ice Accumulation}

By design, LCROSS flew with its solar array pointed toward the sun for most of the mission. Pre-launch analysis anticipated that ice, which had accumulated on the Centaur after fueling on the pad, might remain on the "cold side" of the Centaur for most of flight, and possibly through lunar impact. There was some concern that residual water ice might interfere with

Table 2. Summary of Cold Side Bakeout Maneuvers

\begin{tabular}{|c|c|c|}
\hline Maneuver & Plan Outline & Notes \\
\hline Cold Side Bake 1 & $\begin{array}{l}40 \text { minute roll to } 180^{\circ} ; \\
60 \text { minute dwell } \\
40 \text { minute roll to } 360^{\circ}\end{array}$ & $\begin{array}{l}\text { Expected } \sim 1 \mathrm{~cm} / \mathrm{s} \text { delta-v; } \\
\text { Observed } 3.4 \mathrm{~cm} / \mathrm{s}\end{array}$ \\
\hline Cold Side Bake 2 & $\begin{array}{l}10 \text { minute roll to } 180^{\circ} ; \\
100 \text { minute dwell } \\
10 \text { minute roll to } 360^{\circ}\end{array}$ & $\begin{array}{l}\text { Observed } \sim 1.13 \mathrm{~cm} / \mathrm{s} \text { delta-v; } \\
\text { Significant reduction from } \\
\text { acceleration seen in CSB } 1\end{array}$ \\
\hline Cold Side Bake 3 & $\begin{array}{l}30 \text { minute roll to } 135^{\circ} \\
20 \text { minute dwell } \\
25 \text { minute roll to } 225^{\circ} \text {; } \\
20 \text { minute dwell } \\
30 \text { minute roll to } 360^{\circ}\end{array}$ & $\begin{array}{l}\text { Combined with -Z MGA test; } \\
\text { Similar behavior to CSB 2; } \\
\text { Results inconclusive due to poor } \\
\text { Doppler geometry }\end{array}$ \\
\hline
\end{tabular}
measurements of water ice at the impact site. More significantly, analysis predicted that a sufficient load of water ice on the Centaur exterior, sunlit during its final lunar approach, might push the Centaur off course by hundreds of meters due to sublimation.

Prior to launch, the flight team prepared procedures and command sequences to perform a single "Cold Side Bakeout" maneuver, during which the S-S/C (and Centaur) would be rotated 180 degrees about its longitudinal (roll) axis to face the cold side to the sun as a means of forcing early sublimation of any remaining volatiles. In flight, the execution of this maneuver resulted in an unexpectedly large signature of escaping volatiles (as measured by Doppler shift; see Table 2). A second maneuver exposed the cold side of the Centaur for a longer duration, yet yielded a lesser (but still concerning) signature. A third maneuver seemed to yield similar outgassing, but suffered from poor monitoring geometry. Due to insufficient propellant reserves and time remaining in the mission, the team abandoned further attempts. Pre-impact analysis predicted an impact position error of up to $400 \mathrm{~m}$ (anti-sun direction) due to sublimation; post-impact analysis remains inconclusive.

\section{E. IRU Fault and Excessive Propellant Usage}

On Day 65, upon reacquiring communications with LCROSS after a planned 66 hours out of view, the flight team discovered LCROSS in a backup attitude control configuration. Its IRU was off-line, the star tracker assembly 
(STA) was providing attitude rate information to the ACS, and thrusters were firing nearly continuously. In roughly 24 hours, LCROSS had expended nearly $152 \mathrm{~kg}$ of its remaining $202 \mathrm{~kg}$ of propellant. The project declared an emergency and remained in a full anomaly resolution and recovery posture for two weeks. The propellant loss, it was later determined, was caused by a chain of problems. Roughly 24 hours prior to the anomaly discovery, for reasons not explained (but possibly attributable to a single-event upset), the IRU indicated a fault condition for $5 \mathrm{~ms}$. The fault was detected by ACS fault management and interpreted as an IRU failure. To sustain control, the ACS switched to the STA for attitude rate information. STA-derived rates contained far more noise than those from the IRU, but the ACS was not equipped with STA noise filtering. The noise was interpreted as actual rate information, triggering nearly continuous thruster firing to maintain control. Aside from the short-lived fault condition, the IRU remained fully operational; returning the ACS to IRU-derived rates returned LCROSS to its pre-anomaly ACS propellant consumption rate.

The flight team developed a multi-faceted strategy to protect LCROSS from a similar anomaly as well as from any other anomaly that might cause excessive propellant usage. Onboard fault management was modified to switch to STA backup only in the event of a persistent IRU fault condition. The ACS Stellar Inertial Mode was re-designed to drastically reduce propellant consumption under STA rates, and to be more efficient under IRU rates. To prevent general excessive propellant usage, fault management was also augmented to detect excessive propellant usage, and to respond by transitioning into a modified version of Sun Point Mode (the LCROSS safe mode) that would induce a high yaw rate to make LCROSS passively stable, and then disable attitude control to cease propellant usage.

Following the anomaly, analysis determined that sufficient propellant remained to reliably meet all mission objectives, but only by engaging in propellant-saving measures for the remainder of the mission. Two science calibrations, Earth Look 2 and Moon Look, were removed from the schedule, other activities were merged to take advantage of propellant-saving synergies, and the spacecraft was returned to using paired thruster firings for control (developed originally to counter propellant usage for the Centaur gas leak).

\section{F. Image Compression and Payload Bandwidth}

During the Lunar Swingby Calibration, payload engineers observed that onboard algorithms to compress images of the lunar surface were less effective than during ground-based tests. The team attributed the phenomenon to greater scene complexity in actual images, causing degradation in compression performance. Stemming from this discovery, the Science Team reduced the image sampling frequencies set by impact instrument command sequences, in order to fit within the downlink data rate allowance with worse-than-expected compression ratios. Operationally, contingency ground procedures were developed to quickly update camera sampling rates in near real-time.

During the final lunar approach, image compression performed even less well than for Lunar Swingby. Following from operational modifications, the Science Team made on-the-fly requests to reduce camera sampling rates in the midst of Impact Phase data collection to prevent DHU oversubscription.

\section{Conclusions}

The LCROSS mission was faced with many challenges. Late-breaking predictions of possible Centaur gas leaks forced the operations team to revise or newly develop and test broad-reaching procedures and command sequences in the weeks prior to launch. Strict impact targeting precision requirements were made more challenging under the many perturbations to the LCROSS orbit, including from the spacecraft's own ACS thruster firings and volatiles escaping from the ice-laden Centaur exterior. LCROSS experienced two challenges to propellant reserves - a persistent Centaur gas leak and a propellant-intensive response to a very brief IRU fault. The LCROSS mission operations team, only barely large enough to staff the busy first week of flight, was taxed further by a series of anomalies that, while recoverable, stretched working hours well beyond original expectations.

Despite these hurdles, the LCROSS mission ended successfully. With any space mission, there is a certain amount of luck necessary for success, and LCROSS was no different in that regard. Luck aside, several features of LCROSS spacecraft and mission design contributed directly to mission success. The decision to slip the LRO/LCROSS launch to the June 17 launch block, with its relatively low delta-v requirement (and high propellant margin), was critical to mission success under the IRU fault response anomaly. The large S-S/C power margin, which remained power-positive even under sustained solar array sun angles of 60 degrees, was important to the workaround developed for the thruster thermal anomaly, which was possible only by maintaining a 20 degree sun angle bias for most of the mission. Though the Centaur gas leak was not as serious as some models predicted, the operational workarounds developed prior to launch became central elements of the propellant savings strategy imposed after the IRU anomaly. 
Flight team training and composition also contributed to mission success. With exclusive use of an accurate LCROSS spacecraft simulator for more than a year prior to flight, the flight team trained extensively in nearly every aspect of the LCROSS mission. By launch, the team was largely accustomed to the operational flow and could focus its attention on the unexpected challenges presented by the real spacecraft.

We also attribute the LCROSS success to an array of formal and informal partnerships. When anomalies occurred, the flight team included in its membership numerous Northrop Grumman lead spacecraft subsystem engineers and systems engineers, all of whom had been deeply involved in the design and test of the spacecraft. Strong management relationships built between NASA Ames and Northrop Grumman during spacecraft development continued during flight. As a result of this close partnership, the team transitioned efficiently between nominal and anomaly response postures. Impact targeting benefitted tremendously from the expertise provided by JPL (navigation and link scheduling) and GSFC (maneuver design). On another level, the partnership between LCROSS and LRO teams was extremely valuable to LCROSS. LRO provided critical directed data for impact site selection and supported impact science operations with its observations of the Centaur impact site. Informal partnerships with other mission teams, including Kaguya and Chandrayaan-1, were instrumental in impact site selection. Though never brought into full service, the Goddard Network coordinated with LCROSS in an effort to provide the project with additional communications contact time in the sensitive period after the propellant loss. APL provided valuable tracking data to augment data from the DSN prior to impact. Finally, through the generosity of numerous other missions communicating via the DSN, who sacrificed significant scheduled track time during the IRU fault and subsequent emergency declaration, the LCROSS mission operations team was able to maintain a 24hour communications link to the S-S/C until sufficient fault management products could be developed, tested, and loaded on board.

\section{Appendix A: Acronym List}

\begin{tabular}{|l|l||l|l|}
\hline ACS & attitude control system & LRO & Lunar Reconnaissance Orbiter \\
\hline ARC & NASA Ames Research Center & MCC & mid-course correction (LRO) \\
\hline EDUS & Earth Departure Upper Stage (Centaur) & MIR & Mid-Infrared Camera \\
\hline ESPA & EELV Secondary Payload Adapter & NIR & Near-Infrared Camera \\
\hline DHU & Data Handling Unit & NSP & Near-Infrared Spectrometer \\
\hline DSN & Deep Space Network & S-S/C & LCROSS Shepherding Spacecraft \\
\hline HST & Hubble Space Telescope & STA & star tracker assembly \\
\hline IRU & inertial reference unit & TCM & trajectory correction maneuver (LCROSS) \\
\hline LCROSS & $\begin{array}{l}\text { Lunar CRater Observation and Sensing } \\
\text { Satellite }\end{array}$ & TLI & trans-lunar injection \\
\hline LGALRO & lunar gravity assist lunar return orbit & VSP & Visible Spectrometer \\
\hline LOI & lunar orbit insertion & ULA & United Launch Alliance \\
\hline LPRP & Lunar Precursor Robotic Program & UTC & Universal Coordinated Time \\
\hline
\end{tabular}

\section{Appendix B: Glossary}

\begin{tabular}{|l|l|}
\hline blowdown & $\begin{array}{l}\text { Condition in which propellant is pressurized only by residual propellant in system and } \\
\text { mechanical pressure regulators (e.g. bladder) }\end{array}$ \\
\hline Chandrayaan-1 & $\begin{array}{l}\text { A mission operated by the Indian Space Research Organization (ISRO) to collect global lunar } \\
\text { topographic, chemical, and mineralogical data. Launched 22 October 2008. }\end{array}$ \\
\hline Kaguya & $\begin{array}{l}\text { Known officially as SELENE (Selenelogical and Engineering Explorer), a mission operated by } \\
\text { the Japanese Aerospace Exploration Agency (JAXA) to collect lunar geologic, topographic, } \\
\text { and gravity field data. Launched 14 September 2007. }\end{array}$ \\
\hline periselene & Closest approach to moon \\
\hline
\end{tabular}

\section{Acknowledgments}

This work was funded wholly by NASA under the LCROSS project. The authors wish to thank all those who contributed to the success of the LCROSS mission. 


\section{References}

${ }^{1}$ Feldman, W., et al., "Fluxes of Fast and Epithermal Neutrons from Lunar Prospector: Evidence for Water Ice at the Lunar Poles," Science, Vol. 281, No. 5382, pp. 1496-1500, 4 September 1998.

${ }^{2}$ Chin, G., et al., "Lunar Reconnaissance Orbiter Overview: The Instrument Suite and Mission", Space Science Reviews, Vol. 129, No. 4, 2007, pp. 391-419.

${ }^{3}$ Andrews, D., "LCROSS Lunar Impactor: Pioneering Risk-Tolerant Exploration in a Search for Water on the Moon", Proceedings of the $7^{\text {th }}$ International Planetary Probe Workshop, Barcelona, Spain, June 2010 (submitted for publication).

${ }^{4}$ Goodwin, J. S. and Wegner, P., "Evolved Expendable Launch Vehicle Secondary Payload Adapter: A New Delivery System for Small Satellites," Fifteenth AIAA/USU Conference on Small Satellites, AIAA, Logan, UT, August 2001.

${ }^{5}$ Ennico, K., et al., "LCROSS Science Payload Ground Development, Test and Calibration", 40" Lunar and Planetary Science Conference, Lunar and Planetary Science Institute, No. 1878, The Woodlands, TX, March 2009.

${ }^{6}$ Strong, J., et al., "Transport and Use of a Centaur Second Stage in Space", AIAA Space Ops 2010 Conference, Delivering on the Dream, AIAA, Washington, DC, 2010, Paper 2010-2197 (submitted for publication).

${ }^{7}$ Tompkins, P., et al., "Flight Team Development in Support of LCROSS - A Class D Mission", AIAA Space Ops 2010 Conference, Delivering on the Dream, AIAA, Washington, DC, 2010, Paper 2010-2223 (submitted for publication).

${ }^{8}$ Alena, R., D'Ortenzio, M., Vho, N., "Communication Link Prediction and Assessment for LCROSS Mission Operations", AIAA Space Ops 2010 Conference, Delivering on the Dream, AIAA, Washington D.C., 2010, Paper 2010-1933 (submitted for publication). 